\title{
Downregulation of CD4+CD25+ regulatory T cells may underlie enhanced Th1 immunity caused by immunization with activated autologous $\mathrm{T}$ cells
}

Qi Cao ${ }^{1,2, *}$, Li Wang ${ }^{1,2, *}$, Fang Du ${ }^{1,2}$, Huiming Sheng ${ }^{1,2}$, Yan Zhang ${ }^{1,2}$, Juanjuan $\mathrm{Wu}^{1,2}$, Baihua Shen ${ }^{1,2}$, Tianwei Shen ${ }^{1,2}$, Jingwu Zhang ${ }^{1,2}$, Dangsheng $\mathrm{Li}^{3}$, Ningli $\mathrm{Li}^{1,2}$

${ }^{1}$ Department of Immunology, Shanghai Jiao Tong University School of Medicine, Shanghai, China; ${ }^{2}$ Shanghai Institute of Immunology, 280 South Chongqing Road, Shanghai 200025, China; ${ }^{3}$ Shanghai Institutes for Biological Sciences, Chinese Academy of Sciences, Shanghai, China

Regulatory T cells (Treg) play important roles in immune system homeostasis, and may also be involved in tumor immunotolerance by suppressing Th1 immune response which is involved in anti-tumor immunity. We have previously reported that immunization with attenuated activated autologous $\mathrm{T}$ cells leads to enhanced anti-tumor immunity and upregulated Th1 responses in vivo. However, the underlying molecular mechanisms are not well understood. Here we show that Treg function was significantly downregulated in mice that received immunization of attenuated activated autologous $\mathrm{T}$ cells. We found that Foxp3 expression decreased in CD4+CD25+ T cells from the immunized mice. Moreover, CD4+CD25+Foxp3+ Treg obtained from immunized mice exhibited diminished immunosuppression ability compared to those from naïve mice. Further analysis showed that the serum of immunized mice contains a high level of anti-CD25 antibody (about $30 \mathrm{ng} / \mathrm{ml}, p<0.01 \mathrm{vs}$ controls). Consistent with a role of anti-CD25 response in the downregulation of Treg, adoptive transfer of serum from immunized mice to naïve mice led to a significant decrease in Treg population and function in recipient mice. The triggering of anti-CD25 response in immunized mice can be explained by the fact that CD25 was induced to a high level in the ConA activated autologous T cells used for immunization. Our results demonstrate for the first time that immunization with attenuated activated autologous $\mathrm{T}$ cells evokes anti-CD25 antibody production, which leads to impeded CD4+CD25+Foxp3+ Treg expansion and function in vivo. We suggest that dampened Treg function likely contributes to enhanced Th1 response in immunized mice and is at least part of the mechanism underlying the boosted anti-tumor immunity.

Keywords: immunization with activated autologous T cells, CD4+CD25+Foxp3+ Treg, anti-CD25 antibody, serum adoptive transfer

Cell Research (2007) 17:627-637. doi: 10.1038/cr.2007.46; publication online 12 June 2007

\section{Introduction}

It is well known that CD4+ T cells (also called Th1 cells) play an important role in anti-tumor immune responses [1].

\footnotetext{
*These two authors contributed equally to this work. Correspondence: Ningli Li

Tel: +86-21-64453149; Fax:+86-21-63846383

E-mail: ninglixiaoxue57@yahoo.com.cn

Received 13 December 2006; revised 15 February 2007; accepted 12 March 2007; published online 12 June 2007
}

Th1 cells produce a panel of cytokines called Th1 cytokines including IFN $\gamma$ and TNF $\alpha$. IL-12, produced mainly by monocytes, enhances Th1 immune response [1,2]. Through cytokine productions, Th1 cells synergize with CD8+ T cells (called CTL) in their anti-tumor function. For example, IFN $\gamma$ can activate CTL, which promote tumor cell lysis by secreting perforin, granzyme B and TNFa $[3,4]$. TNF $\alpha$ can induce dendritic cells (DC) mediated tumor antigen recognition $[5,6]$. IL-12 is a key cytokine to induce Th1 cell differentiation and enhance Th1 function $[7,8]$. Many reports have shown that activity of Th1 
cells is downregulated in patients with malignant tumors. For example, tumor patients had lower concentrations of Th1 cytokines in serum, which may lead to low CTLmediated anti-tumor activity and dampened DC function [9-11]. Treating patients with IL-2 plus IFN $\gamma$ may induce tumor regression $[12,13]$. Therefore, Thl cytokines are believed to be an effective adjuvant immunotherapy in tumor treatment [14].

It is indicated that malignant tumor cells may induce immune tolerance by multiple mechanisms. For example, some tumors do not express major histocompatibility complex (MHC) molecules so that these tumor cells do not elicit sufficient anti-tumor immunity $[15,16]$. In other cases, tumor cells fail to generate new antigen or tumor-specific antigens; and as a result they are unable to evoke immune recognition and tumor destruction because of low intrinsic immunogenicity and tumor-associated immunosuppression $[17,18]$. Moreover, even when tumor cells express tumor antigens which may act as an efficient target for MHC class I-restricted responses in vivo, the immune system still fails to produce an effective anti-tumor immune response [19, 20]. The pathological interactions between cancer cells and host immune cells in the tumor microenvironment create an immunosuppressive network that promotes tumor growth, protects the tumor from immune attack and attenuates immunotherapeutic efficacy [21,22]. All in all, malignant tumor cells may induce tolerance in the bodies bearing tumors [23].

Tolerance is the 'holy grail' in the field of immunology and is generally divided into central tolerance and peripheral tolerance. Regulatory (or previously called suppressor) T cells (Treg) are naturally occurring or inducible lymphocytes that have been shown to downmodulate a series of immune responses. Although it is now clear that the phrase 'regulatory T cells' encompasses more than one cell type, CD4+CD25+ Foxp3+ Treg cells have received attention due to their immunosuppressive properties in vitro and in vivo [14, 24, 25]. It has been suggested that Treg are responsible for inducing and maintaining peripheral tolerance and for negative regulation of tumor immunity so that they contribute to tumor growth in mice $[12,13,26]$. Curiel et al. [27] presented evidence that human Treg have an important immunopathological role in human cancer by suppressing tumor-associated-antigen-specific T-cell immunity. Because a number of studies have demonstrated that Treg-mediated immunosuppression is one of the crucial mechanisms of tumor immune-evasion and the main obstacle of successful tumor immunotherapy [14, 27-29], Treg depletion has been attempted in tumor therapy. While CTLA-4 blockade has been shown to enhance anti-tumor immunity [30,31], a number of studies have implicated that Treg depletion could be another effective strategy in tumor biotherapy. For example, Onizuka et al. [32] and Shimizu et al. [33] reported that a single in vivo administration of anti-CD25 monoclonal antibody caused the regression of tumors that grew progressively in syngeneic mice. Sutmuller et al. [34] showed that CTLA-4 blockade and Treg depletion could synergize to elicit enhanced therapeutic anti-tumor immunity.

In our previous work, we reported that administration with irradiated, activated autologous $\mathrm{T}$ cells in mice evokes anti-tumor immunity by enhancing the survival of activating $\mathrm{T}$ cells, which is accompanied by GADD $45 \beta$ upregulation in T cells [35]. In this report, we further investigated the anti-tumor mechanisms elicited by activated autologous $\mathrm{T}$ immunization. We unexpectedly found that CD4+CD25+ T cells were downregulated by our immunization procedure. This downregulated population was further identified as CD4+CD25+Foxp3+ Treg by Foxp3 staining and proliferation-inhibition assay. Interestingly, we found that downregulation of Treg after immunization was caused by anti-CD25 antibody produced in immunized mice. We thus demonstrated for first time that immunization with irradiated, activated autologous $\mathrm{T}$ cells could induce anti-CD25 antibody production in recipient mice, which leads to downregulation of both the population and suppressor function of Treg. We suggest that reduced Treg function induced by our immunization might relieve Th1 from suppression and allow for production of more cytokines, which in turn contributes to enhanced anti-tumor immunity in vivo.

\section{Materials and Methods}

\section{Mice}

Female C57/Bl/6 mice were purchased from the Shanghai Laboratory Animal Center, Chinese Academy of Sciences. Mice were maintained under pathogen-free conditions and used between ages of 6 and 8 weeks.

\section{Autologous activated T-cell preparation and mice adminis- tration}

Splenocytes $\left(5 \times 10^{6} / \mathrm{ml}\right)$ were purified and stimulated with $5 \mu \mathrm{g} / \mathrm{ml}$ ConA (Vector Laboratories, Burlingama, CA, USA) in complete DMEM (DMEM with 10\% fetal calf serum, $10 \mathrm{mM}$ HEPES, 50 $\mu \mathrm{M} \beta$-mercaptoethanol, $2 \mathrm{mM}$ L-glutamine and $50 \mathrm{IU} / \mathrm{ml}$ penicillinstreptomycin) in $25 \mathrm{ml}$ flasks for $72 \mathrm{~h}$. The cells were collected and washed three times with PBS ( $\mathrm{pH} 7.4$ ). T cells were then purified using Dynabeads coated with specific antibodies by negative selection (Dynal Biotech, Oslo, Norway). The purity of T cells obtained from culture was greater than $92 \%$. Purified T cells were irradiated at 3 $000 \mathrm{rad}$, and mice were administrated for immunization as described in our previous report [35].

\section{Cytokine measurement by ELISA}

Sera were collected from both immunized mice and naïve mice before they were sacrificed. The serum samples were tested in 
duplicate for production of IFN $\gamma$, IL-12, TNF $\alpha$ and IL-10 using enzyme-linked immunosorbent assay (ELISA) kits, according to the manufacturer's instructions (R\&D, Minneapolis, MN, USA). Briefly, microtiter plates were coated overnight at $4{ }^{\circ} \mathrm{C}$ with monoclonal anti-mouse IFN $\gamma$, IL-12, TNF $\alpha$ and IL-10 antibodies respectively (capturing antibody). Wells were then blocked at room temperature for $2 \mathrm{~h}$ with PBS containing 3\% bovine serum albumin (BSA) (Sigma) and washed four times with PBS containing $0.02 \%$ Tween20. After the wash, the sera collected from mice were added. The plates were incubated at $4{ }^{\circ} \mathrm{C}$ overnight followed by four additional washes. Appropriate biotinylated detecting antibodies $(0.25 \mu \mathrm{g} / \mathrm{ml})$ were added and incubated at RT for $2 \mathrm{~h}$. Plates were washed four times and incubated with avidin-conjugated horseradish peroxidase at 1:2000 dilution. $0.0123 \%$ tetramethylbenzidine $/ 0.008 \% \mathrm{H}_{2} \mathrm{O}_{2}$ in citrate buffer ( $\mathrm{pH}$ 5.0) was used as a substrate. After 20 min incubation at room temperature, the color development was stopped using $1 \mathrm{M} \mathrm{H}_{2} \mathrm{SO}_{4}$. Optical densities were measured using an ELISA reader at $450 \mathrm{~nm}$ and the concentration of cytokine was calculated.

Isolation of $C D 4+, C D 4+C D 25-$, and CD4+CD25+ T cells

Fresh spleens were removed and prepared for single-cell suspensions. CD4+ T cells were negative isolated from the resulting splenocytes using magnetic bead separation. Briefly, splenocytes were depleted for CD8+, B220+, CD16+, Gr-1+ and Ly76+ cells using biotin-labeled specific mAb (BD Biosciences PharMingen, San Diego, CA, USA), anti-biotin magnetic beads, and an LD magnetic bead column (Miltenyi Biotec, Auburn, CA, USA). To isolate CD4+CD25+ or CD4+CD25- T cells, purified CD4+ T-cell populations were incubated with biotin-labeled anti-CD25 antibody (BD Biosciences) and anti-biotin magnetic beads and were isolated by MACS separation column (Miltenyi Biotec). To isolate mouse CD4+CD25- T cells, CD4+ T cells were first purified from splenocytes using a CD4+ No-touch T-cell isolation kit (Miltenyi Biotec, Auburn, CA, USA) and CD4+CD25- T cells were isolated by negative selection using CD25 microbeads (Miltenyi Biotec). The purity of CD4+, CD4+CD25+ or CD4+CD25-T-cell fractions was always greater than $95 \%$.

\section{Cell surface molecules and Foxp3 analysis by flow cytom-} etry

Cells activated by ConA were used for mice immunization, and single splenocytes were obtained from immunized and naïve mice, and were stained with special goat anti-mouse CD3, CD4, CD8, CD25, CD62L and CD69 antibodies followed by flow cytometry detection. Briefly, cells were resuspended in PBS containing 1\% BSA (Irvine) and $0.1 \%$ sodium azide (Sigma-Aldrich). For surface staining of CD4, CD8, CD25, CD62L and CD69, T cells were incubated with fluorochrome-conjugated antibodies to the indicated cell surface markers (eBioscience) at the recommended dilution or the isotype control antibodies for $30 \mathrm{~min}$ on ice. For intracellular staining of Foxp3, cells were fixed and permeablized with Foxp3 staining buffer (eBioscience). Resulting cells were stained with PE-conjugated anti-mouse Foxp3 mAb $\left(0.5 \mu \mathrm{g} / 10^{6}\right.$ cells; eBiosceience). Stained cells were analyzed subsequently using a FACS Calibur ${ }^{\mathrm{TM}}$ (Becton Dickinson, San Jose, CA, USA).

\section{Proliferation and inhibition assays}

In proliferation assays, mouse splenocytes $\left(2 \times 10^{5}\right.$ per well $)$ were cultured in triplicate in complete DMEM (DMEM with $10 \%$ fetal calf serum, HEPES, $\beta$-mercaptoethanol, L-glutamine, sodium pyruvate and penicillin/streptomycin) in 96-well flat-bottomed plates. Cells were cultured in the presence or absence of ConA $(5 \mu \mathrm{g} / \mathrm{ml})$ at 37 ${ }^{\circ} \mathrm{C}$ in $5 \% \mathrm{CO}_{2}$ for $72 \mathrm{~h}$. Cells were pulsed with $1 \mu \mathrm{Ci}\left[{ }^{3} \mathrm{H}\right]$-thymidine during the last $16-18 \mathrm{~h}$ of culture before harvest. $\left[{ }^{3} \mathrm{H}\right]$-thymidine incorporation was measured as CPM using a $\beta$-plate counter. To evaluate the inhibitory activity of Treg, freshly isolated CD4+CD25- T cells from naïve mice were used as the responder. Antigen presenting cells (APCs) were obtained by isolating the positive fraction after removal of the CD4+ cells from splenocytes by magnetic sort, and were irradiated with $5000 \mathrm{rad} .2 \times 10^{4} \mathrm{CD} 4+\mathrm{CD} 25-\mathrm{T}$ cells were stimulated with ConA $(5 \mu \mathrm{g} / \mathrm{ml})$ in the presence of $1 \times 10^{5}$ irradiated APCs with or without the addition of CD4+CD25+ T cells (from naïve or immunized mice respectively, at the cell density of $2 \times 10^{4} /$ well). The ability of CD4+CD25+ T cells (inhibitor) to suppress the proliferation of CD4+CD25-T cells (responder) was determined by $\left[{ }^{3} \mathrm{H}\right]$-thymidine incorporation. In each case, the percentage of inhibition on proliferation was calculated as [1- (experimental CPM/control $\mathrm{CPM})] \times 100 \%$, where experimental and control CPM refer to the counts with and without the addition of Treg, respectively.

\section{RNA extraction and Foxp3 expression analysis by real-time $P C R$}

Total RNA was isolated from cell pellets using RNeasy Mini Kit (Qiagen, Hilden, Germany). Genomic DNA was removed from total RNA before cDNA synthesis using the RNase-free DNase Set for DNase digestion during RNA purification (Qiagen). RNA was stored at $-80^{\circ} \mathrm{C}$. First-strand cDNA synthesis was performed for each RNA sample using Sensiscript RT Kit (Qiagen). Random hexamers (BioAsia Biotechnology, Shanghai, China) were used to prime cDNA synthesis. mRNA expression level of Foxp3 was determined by real-time PCR using SYBR Green master mix (Applied Biosystems, Foster City, CA, USA). Thermocycler conditions comprised an initial holding at $50{ }^{\circ} \mathrm{C}$ for $2 \mathrm{~min}$, then $95{ }^{\circ} \mathrm{C}$ for 10 min, which was followed by a two-step PCR program consisting of $95^{\circ} \mathrm{C}$ for $15 \mathrm{~s}$, and $60{ }^{\circ} \mathrm{C}$ for $60 \mathrm{~s}$ for 40 cycles. Data were collected and quantitatively analyzed on an ABI Prism 7900 sequence detection system (Applied Biosystems). The mouse $\beta$-actin gene was used as an internal control to normalize for differences in the amount of total RNA in each sample. All quantities were expressed as number of folds relative to the expression of $\beta$-actin. PCR primer pairs were as follows: 5'-AGG AGC CGC AAG CTAAAA GC-3' and 5'-TGC CTT CGT GCC CAC TGT-3' for mouse FoxP3; 5'-TGT CCA CCT TCC AGC AGA TGT-3' and 5'-AGC TCA GTA ACA GT C CGC CTA G A-3' for mouse $\beta$-actin.

\section{Measuring serum level of anti-mouse CD25 antibody}

Sera were collected from both immunized mice and naïve mice before they were sacrificed. The serum samples were tested in duplicate for production of anti-CD25 antibody using ELISA. Briefly, microtiter plates were coated overnight at $4{ }^{\circ} \mathrm{C}$ with $50 \mathrm{ng} /$ well of purified mouse CD25 protein (R\&D, Minneapolis, MN, USA). After blocking at room temperature for $2 \mathrm{~h}$ with PBS containing $3 \%$ bovine serum albumin (BSA) (Sigma) followed by washing four times with PBS containing $0.02 \%$ Tween-20, the sera collected from mice were added. As a positive control, different amounts of a specific monoclonal anti-mouse CD25 antibody (eBioscience, USA) were added into the coated wells to generate a standard curve. The plates were incubated at $4{ }^{\circ} \mathrm{C}$ overnight followed by four additional 
washes. Then appropriate biotinylated-detecting antibody $(0.25 \mu \mathrm{g} /$ $\mathrm{ml}$ ) was added and incubated at RT for $2 \mathrm{~h}$. Plates were washed and incubated with avidin-conjugated horseradish peroxidase at 1:2000 dilution. $0.0123 \%$ tetramethylbenzidine $/ 0.008 \% \mathrm{H}_{2} \mathrm{O}_{2}$ in citrate buffer (pH 5.0) was used as a substrate. After 20 min incubation at room temperature, the color development was stopped using $1 \mathrm{M}$ $\mathrm{H}_{2} \mathrm{SO}_{4}$. Optical densities were measured using an ELISA reader at $450 \mathrm{~nm}$ and concentrations of anti-CD25 were calculated according to the standard curve.

Serum adoptive transfer and assay for the inhibition function of Treg

Sera $(0.8 \mathrm{ml})$ isolated from naïve and immunized mice respectively were transferred into naïve mice by invtravenous injection (i.v.). The CD4+CD25+ T cells in peripheral blood mononuclear cells (PBMC) of recipient mice were measured every day for 4 days after the transfer. CD4+T cells were isolated from splenocytes obtained from recipient mice as described in earlier sections. Foxp3 staining and analysis for the inhibition function of CD4+CD25+ T cells were carried out as described above.

\section{Statistical analysis}

Student's $t$-test was used to analyze the differences between the groups. One-way ANOVA was initially performed to determine whether an overall statistically significant change existed before using the two-tailed paired or unpaired Student's $t$-test. $p$-value of $<0.05$ was considered statistically significant.

\section{Results}

Enhancement of IFN $\gamma$ and IL-12 production in immunized mice

As shown in our previous work, immunization with attenuated activated autologous T cells evokes anti-tumor

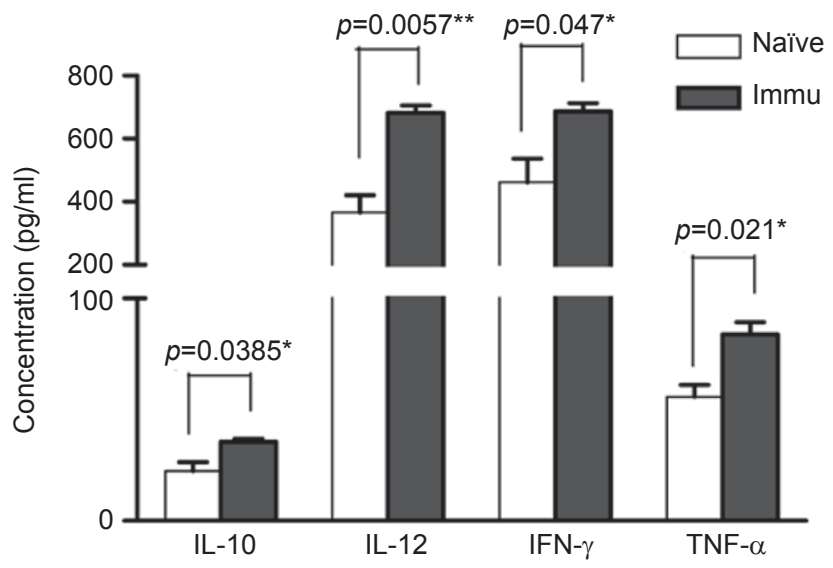

Figure 1 Enhancement of Th1 cytokine and IL-12 production in immunized mice. Sera were colleted from both naïve (without immunization) and immunized C57 mice (immunization with attenuated activated autologous T cells). Levels of IL-12, IFN $\gamma$, TNF $\alpha$ and IL-10 in serum isolated from both immunized and naive mice were quantified by ELISA. Open bars represent samples from naïve mice while black bars represent samples from immunized mice $(n=$ 4 in each group of experiments). The data represented one of three experiments. immunity by inducing IL-12 production and CTL-mediated cytotoxicity. To analyze CD4+ T-cell function after immunization, a panel of cytokines in immunized mice was quantified and compared with those from naïve mice. The sera were collected from both immunized mice and
A

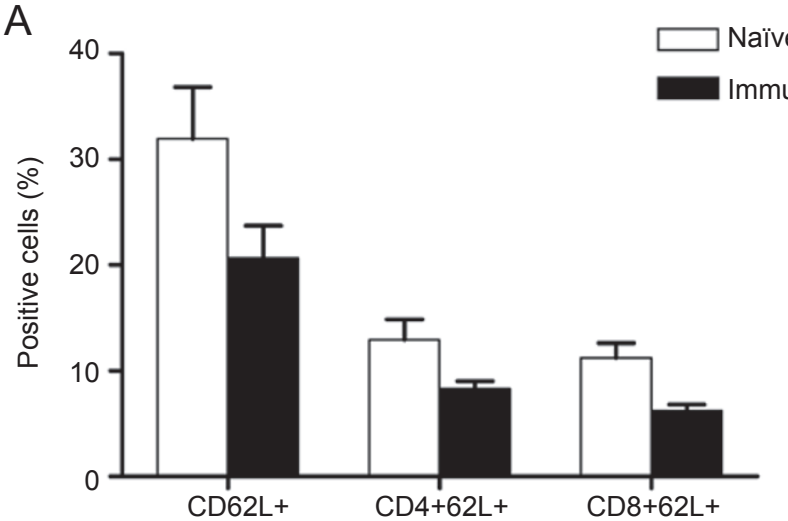

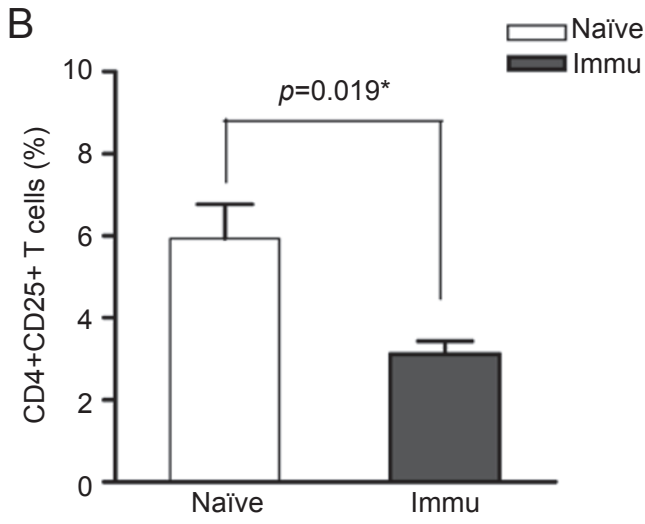

Figure 2 T-cell activation profile in vivo induced by autologous T-cell immunization. The PBMC freshly isolated from naïve and immunized mice were stained with goat anti-mouse CD3, CD4 and CD8. For T-cell activation analysis, anti-CD25 and CD62L antibodies were used to detect CD25 and CD62L expression on CD4+ T cells by flow cytometry. The open and black bars represent the cells isolated from naive and immunized mice respectively in all panels. (A) Assay for resting T cells using anti-CD62L antibody staining $(n=4)$. (B) Assay for CD25+ cells using anti-CD25 staining $(n=4)$. The data represented one of three experiments. 
naïve mice before they were sacrificed. The serum samples were tested in duplicate for the production of IFN $\gamma$, IL-12, TNF $\alpha$ and IL-10 using ELISA kits (R\&D). In this assay, the IL-12 level was significantly increased in the immunized mice as we reported before $(p=0.0057)$ [35]. Th1 cytokines IFN $\gamma$ and TNF $\alpha$ increased significantly also in immunized mice than those from naïve mice $(p=0.047$ and 0.021 respectively). IL-10, a Th2 cytokine, was also increased in immunized mice but its absolute concentration (around $35 \mathrm{ng} / \mathrm{ml}$ ) was lower compared with IL-12 and the two Th1 cytokines. For example, IFN $\gamma$ in immunized mice was $700 \mathrm{ng} / \mathrm{ml}$ compared with $400 \mathrm{ng} / \mathrm{ml}$ in naïve mice $(p<0.001)$. Similarly, concentration of IL-12 in immunized mice was $700 \mathrm{ng} / \mathrm{ml}$, but in naive mice the concentration of IL-12 was $300 \mathrm{ng} / \mathrm{ml}(p<0.05)$. These results suggested that the immunization induced both IL-12 and INF $\gamma$ production (Figure 1), which in turn might favor Th1 response and anti-tumor immunity.

\section{Reduced CD4+CD25+ T-cell population in immunized mice}

We showed previously that immunization with attenuated activated autologous $\mathrm{T}$ cells triggers T-cell activation in vivo [35]. We tried to address the T-cell activation pattern induced by our immunization. CD62L was used to detect resting T-cell population and CD25 was used to detect activating T cells respectively [12-14]. For this assay, the PBMC were collected, and CD62L and CD25 on CD4+ and CD8+ T cells in PBMCs were identified by flow cytometery. The results showed that the $\mathrm{CD} 62 \mathrm{~L}+\mathrm{CD} 4+\mathrm{T}$ and $\mathrm{CD} 62 \mathrm{~L}+\mathrm{CD} 8+\mathrm{T}$ cells were lower in mice that received three times of immunization than that in naïve mice (Figure 2A). This result suggests that there were fewer resting $T$ cells in the peripheral $\mathrm{T}$ population after immunization. Intriguingly, in this assay we unexpectedly found that within CD4+ T cells the number of CD25+ cells also did not increase, as the $\mathrm{CD} 4+\mathrm{CD} 25+\mathrm{T}$ population decreased significantly in immunized mice ( $p=0.019$; Figure $2 \mathrm{~B}$ ). This implicated that immunization with irradiated autologous $\mathrm{T}$ cells did not increase $\mathrm{CD} 4+\mathrm{CD} 25+\mathrm{T}$ population. It is well known that $\mathrm{CD} 25$ serves as a dual marker in $\mathrm{CD} 4+\mathrm{T}$ cells. CD25 is expressed on activated CD4+ T cells and recently is also used as a cell surface marker for Treg. Since $\mathrm{CD} 4+\mathrm{CD} 25+\mathrm{T}$ are a heterogeneous population consisting of activated CD4+ T cells and Treg, we further asked which sub-population of $\mathrm{CD} 4+\mathrm{T}$ cells were diminished.

\section{Downregulation of regulatory T-cell population in im- munized mice}

As we failed to detect an increase of circulated $\mathrm{CD} 4+\mathrm{CD} 25+\mathrm{T}$ cells in immunized mice, we asked whether the reduced population of CD4+CD25+ T cells represent
A

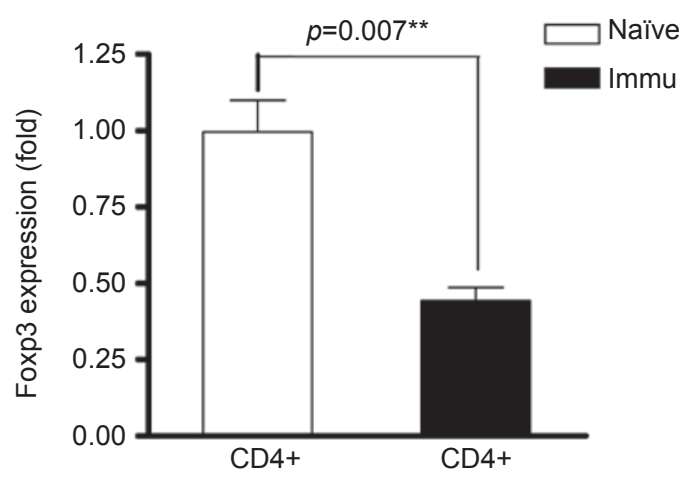

B

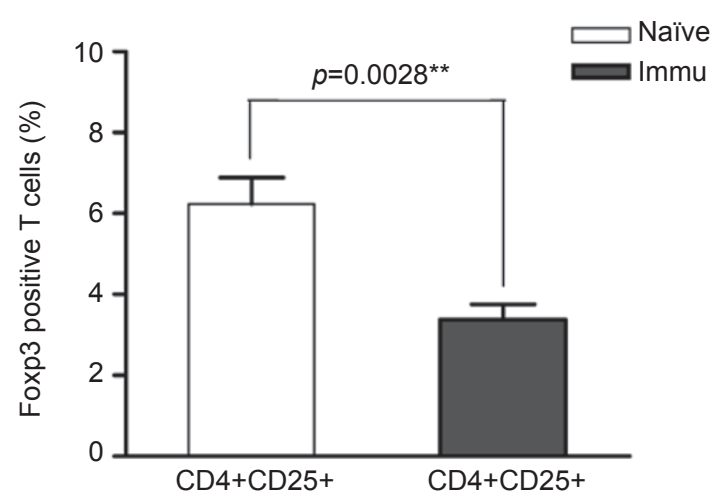

C

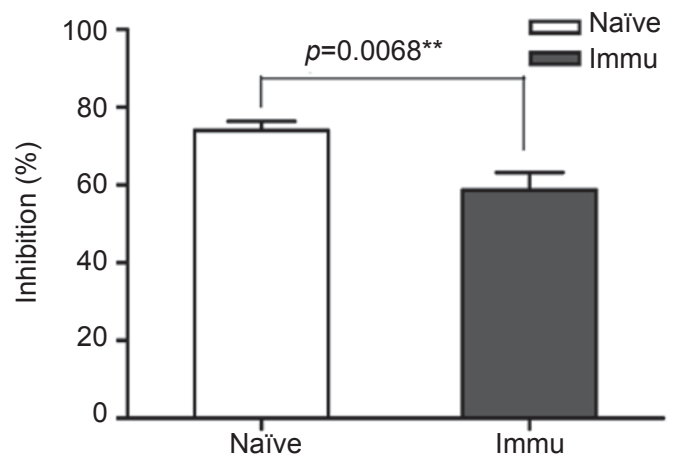

Figure 3 Immunization reduced the population and inhibition function of CD4+CD25+Foxp3+ T cells. Foxp3 expression in CD4+T cells freshly obtained from immunized or naive mice was quantitatively analyzed by both real-time PCR. For real-time PCR assay, the total RNA was extracted and mRNA level of Foxp3 was analyzed and normalized to the internal control ( $\beta$-actin) in the same sample. Relative expression was calculated as the difference $(\Delta \Delta C T)$ between the $\Delta \mathrm{CT}$ values of immunized mice and that of naïve mice, and expressed as $2^{-\Delta \Delta C T}$. For analysis of Foxp3 protein expression, freshly isolated splenocytes were triple stained with antibodies against CD4, CD25 and Foxp3 as described in Materials and Methods. Stained cells were then analyzed by flow cytometry. The inhibition function of CD4+CD25+ T cells was examined as described in Materials and Methods. The open and black bars represent cells isolated from naive and immunized mice, respectively, in all three panels. (A) Relative expression of Foxp3 in CD4+ T cells isolated from naïve and immunized mice $(n=4)$. (B) Foxp3 protein expression in CD4+CD25+T cells $(n=6)$. (C) CD4+CD25+ T cell-mediated inhibition of the proliferation of CD4+CD25-T cells $(n=6)$. The data represented one of three experiments. 
Treg. We examined the expression of Foxp3, a pivotal Treg marker, in the CD4+CD25+ T cells by real-time PCR and intracellular staining. The results indicated that the CD4+CD25+ Foxp3+ T cells were markedly decreased in immunized mice as examined by simultaneous staining with specific anti-CD4, CD25 and Foxp3 monoclonal antibodies and flow cytometery. The results showed that Foxp3 expression was significantly lower in CD4+ T cells from immunized mice compared to naïve mice ( $p=0.007$; Figure $3 \mathrm{~A})$. The Foxp $3+$ population was reduced in $\mathrm{CD} 4+\mathrm{CD} 25+\mathrm{T}$ cells isolated from immunized mice compared with that in naïve mice ( $p=0.0028$; Figure 3B). These results implicated that immunization with autologous activated $\mathrm{T}$ cells induced a drop in Treg population in vivo. To clarify whether Treg function was impaired in immunized mice, the ability of these Treg to inhibit the proliferation of CD4+CD25- T cells was studied. CD4+CD25-T cells were purified from normal naive $\mathrm{C} 57$ mice as responders, and CD4+CD25+ T cells derived from immunized mice and their counterparts obtained from naïve mice were used as inhibitors. Cell proliferation was measured by thymidine incorporation. The results showed that Treg isolated from immunized mice were impaired in their ability to inhibit the proliferation of CD4+CD25- T cells, while the proliferation was inhibited by Treg from normal naive mice ( $p=0.0068$; Figure $3 \mathrm{C}$ ). These data strongly suggest that immunization with irradiated activated autologous $\mathrm{T}$ cells impairs the regulatory function of CD4+CD25+ Treg cells in vivo.

Immunization with autologous activated $T$ cells induces anti-CD25 antibody in vivo

To address how and why immunization reduced the population and regulatory function of CD4+CD25+ Treg, we asked whether there was anti-CD25 production in sera from immunized mice. To test this, we first quantified B cell population with specific anti-B220 staining. The results indicated that $\mathrm{B} 220$ positive cells were markedly increased in immunized mice ( $p<0.0001$; Figure 4A). Further IgGbinding profile was scanned with flow cytometery and the results showed that the IgG in the sera collected from immunized mice bound specifically with ConA activated autologous $\mathrm{T}$ cells used for immunization (Figure 4B). To address whether this kind of reactive IgG from immunized mice represents anti-CD25 antibody, we performed ELISA analysis. The results showed that anti-CD25 antibody was detected from immunized mice using $50 \mathrm{ng} / \mathrm{ml}$ of commercial purified mouse CD25 to coat the plates, and that the anti-CD25 antibody was dramatically increased in the sera from immunized mice. The concentration of anti-CD25 was quantitatively analyzed using the commercial purified anti-CD25 monoclonal antibody as the standard curve, and the results showed that the level of specific anti-CD25 an-
A

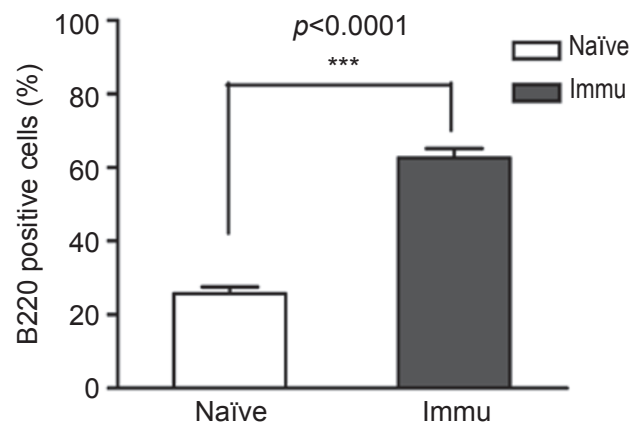

B

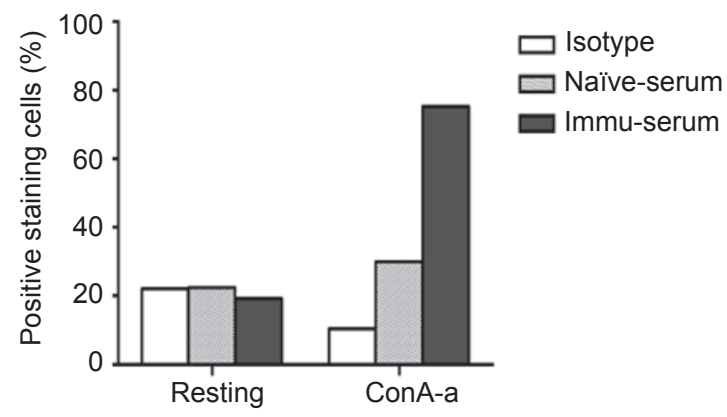

C

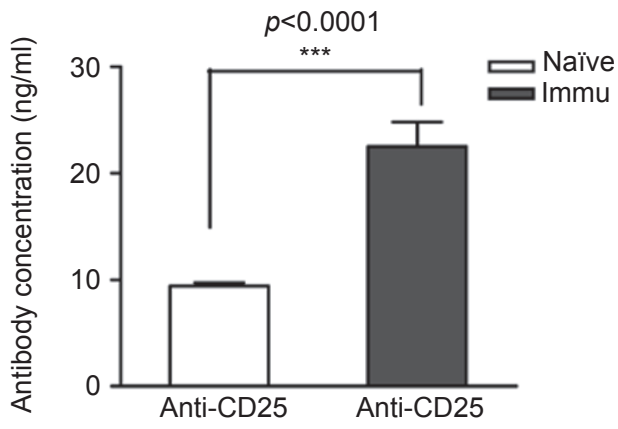

Figure 4 Immunization with autologous-activated T cells induced anti-CD25 antibody production in vivo. Single splenocytes obtained from naïve and immunized mice were stained by anti-B220 antibody for $\mathrm{B}$ cell population. ConA-activated $\mathrm{T}$ cells used for immunization and resting cells were stained by serum collected from both naïve and immunized mice, followed by staining with goat-anti mouse IgG and examined by flow cytometry. The levels of anti-CD25 antibody in serum collected from naive and immunized mice were quantified by ELISA as described in Materials and Methods. (A) B220 expression pattern in naïve and immunized mice. The open and black bars represent cells isolated from naive and immunized mice respectively $(n=6)$. (B) Staining patterns of resting and ConA activated T cells. The open bars represent IgG isotype control. The hatched and black bars represent staining with serum of naïve and immunized mice respectively. (C) Quantitative analysis of anti-CD25 antibody levels in sera of naïve (open bar) and immunized (black bar) mice $(n=12)$. 
tibody in immunized mice was $28.3 \pm 1.8 \mathrm{ng} / \mathrm{ml}$ compared to $8.7 \pm 1.2 \mathrm{ng} / \mathrm{ml}$ in the sera collected from naïve mice $(p<$ 0.0001 ; Figure 4C). These data implicated that there were higher levels of anti-CD25 antibody induced in the immunized mice, which might be responsible for the reduced Treg population and impaired Treg function in vivo.

\section{CD25 was dominantly expressed on activated autologous} T cells used for immunization

To address how and why immunization induced antiCD25 antibody in vivo, the kinetic expressions of CD62L, CD69 and CD25 which represent different activation stages of $T$ cells were investigated. T cells were collected and stained by specific antibodies followed by cytometery detection at every $24 \mathrm{~h}$ after stimulation with ConA. The results showed that these three molecules displayed different expression pattern on the cells with different peak point. At $72 \mathrm{~h}$, both CD62L and CD69 showed low expression on cell surface; even though they did increase at $48 \mathrm{~h}$ after stimulation by ConA, their levels dropped quickly afterwards and returned to the base line at $72 \mathrm{~h}$. In contrast, CD25 expression increased continuously in a time-dependent manner when the cells were incubated with ConA (Figure $5 \mathrm{~A})$. It is worth to point out that about $70 \%$ of CD3+T cells expressed CD25 molecule while only a small portion of them expressed CD62L and CD69 at $72 \mathrm{~h}$ after stimulation with ConA(Figure 5B). This implicated that CD25 was the dominant molecule expressed on the ConA activated T cells that were used for immunization. The molecule profiles on cells used for immunization suggested that the activated autologous $\mathrm{T}$ cells used for immunization might bring abundant CD25 molecules into the immunized mice, and these CD25 molecules might stimulate the recipient mice to produce anti-CD25 antibody, which in turn reduces the population and function of Treg in these mice.

\section{Adoptive transfer of sera from immunized mice resulted in} Treg reduction in recipient mice

To address whether the reduction of Treg population and function in immunized mice was due to the higher level of anti-CD25 antibody induced by immunization, serum adoptive transfer assay was performed. Sera were collected from both naïve and immunized mice, and 0.8 $\mathrm{ml}$ of sera were injected into naïve mice by i.v. Following sera adoptive transfer, the kinetics of CD4+CD25+ T cells in PBMC from the recipient mice were measured every day for 4 days. The results demonstrated that $\mathrm{CD} 4+\mathrm{CD} 25+\mathrm{T}$ cells were decreased at day 1 after sera transfer and reached the lowest level on day 2. On day 3, the CD4+CD25+ T cells recovered and returned to the original level by day 4 . Compared with mice that received sera from naïve mice, $\mathrm{CD} 4+\mathrm{CD} 25+\mathrm{T}$ cells were markedly reduced after receiving sera from immunized mice especially on day $2(p=0.03$; Figure 6A). We also analyzed Foxp3 expression in CD4+T cells in the recipient mice and examined Treg-mediated inhibition function. The results showed that Foxp3 expression was reduced in $\mathrm{CD} 4+\mathrm{T}$ cells obtained from immunization sera recipients compared with those naïve sera recipients ( $p=0.0037$; Figure 6B). Meanwhile, the inhibitory activity assay showed that the suppressive function of $\mathrm{CD} 4+\mathrm{CD} 25+\mathrm{T}$
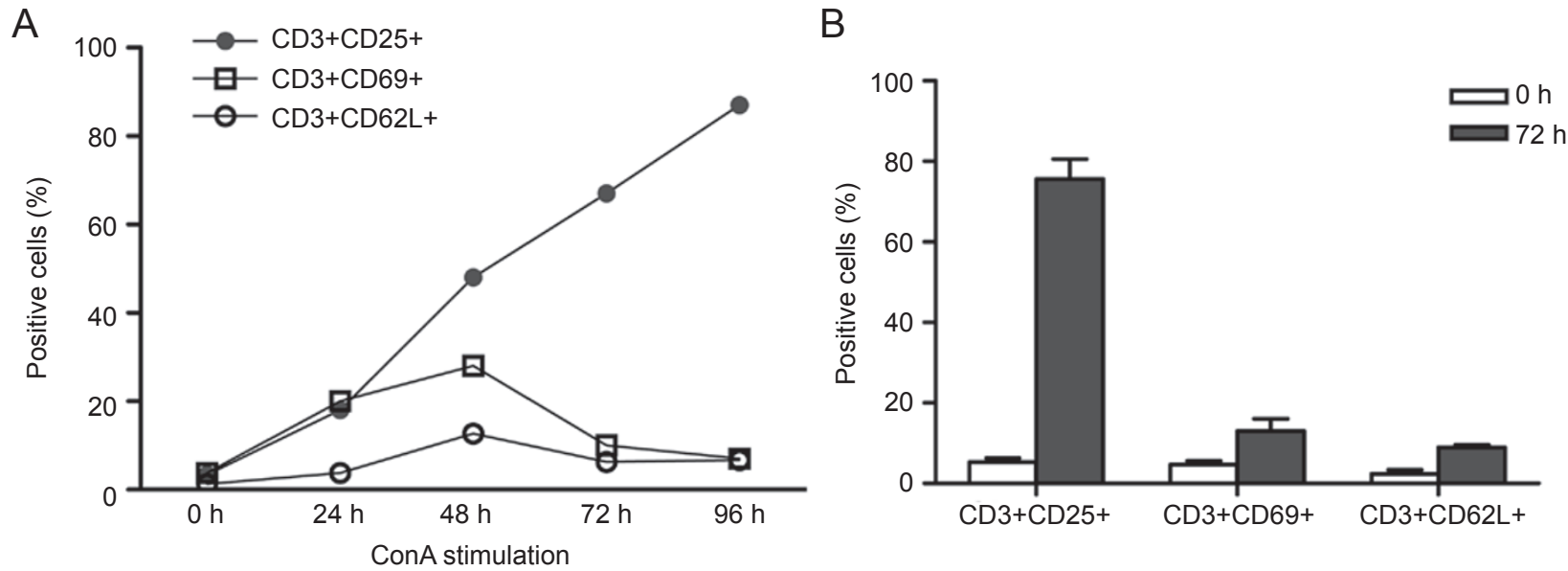

Figure 5 Dominant expression of CD25 on activated autologous T cells used for immunization. Expression of CD25, CD62L and CD69 on ConA activated autologous T cells used for immunization was quantified by specific antibodies and detected by flow cytometry. (A) Kinetic expression of CD25, CD62L and CD69 on ConA activated T cells. (B) Expression of CD25, CD62L and CD69 on $\mathrm{T}$ cells at $72 \mathrm{~h}$ after ConA stimulation $(n=5)$. 
A

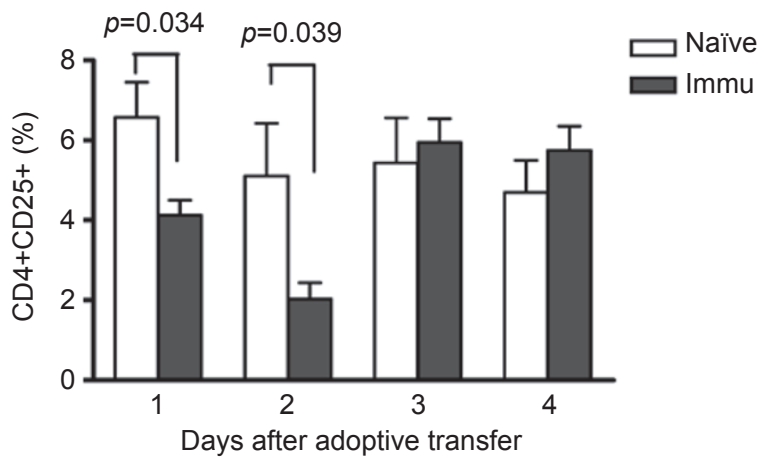

B

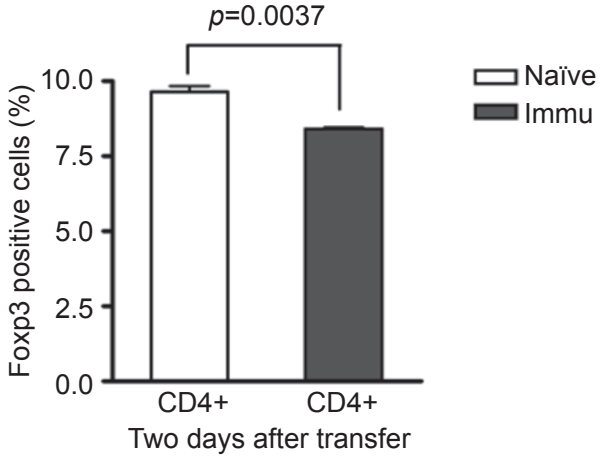

C

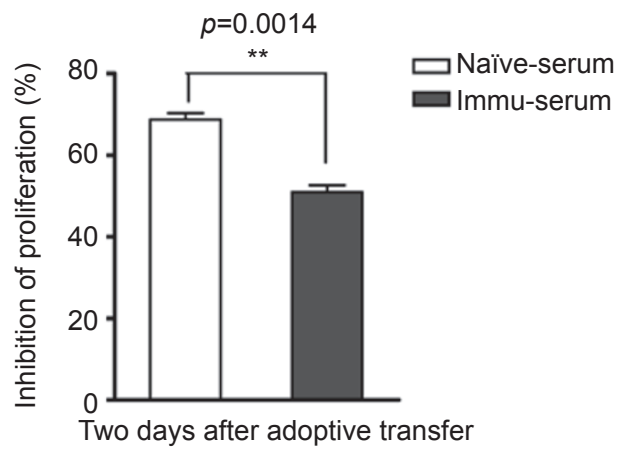

Figure 6 Adoptive transfer with sera obtained from immunized mice downregulated $\mathrm{CD} 4+\mathrm{CD} 25+$ Foxp $3+$ Treg population and function in recipient mice. Sera were collected from naïve and immunized mice respectively and transferred into naïve mice by i.v. $\mathrm{CD} 4+\mathrm{CD} 25+\mathrm{T}$ cells in recipient mice were detected by flow cytometry every day for 4 days after the transfer. The percentage of Foxp $3+$ cells within $\mathrm{CD} 4+\mathrm{T}$ cells in the recipient mice at day 2 after the transfer was analyzed by flow cytometry. CD4+CD25+ T cells isolated from the recipient mice at day 2 were examined for their ability to inhibit the proliferation of CD4+CD25-responder cells as described in Materials and Methods. (A) The profiles of CD4+CD25+ T population in PBMC of recipient mice at different days after the sera transfer $(n=$ 4). (B) Foxp3 expression in the CD4+CD25+ T cells obtained from recipient mice at day $2(n=3)$. (C) The inhibition of responder cell proliferation by $\mathrm{CD} 4+\mathrm{CD} 25+\mathrm{T}$ cells obtained from recipient mice at day 2 after the sera transfer $(n=3)$. The data represented one of three experiments. cells isolated from mice that received sera of immunized mice was significantly decreased ( $p=0.0014$; Figure 6C). These data suggest that anti-CD25 antibody induced by immunization mediated Treg downregulation in vivo.

\section{Discussion}

We have previously reported that immunization with attenuated activated autologous $T$ cells leads to enhanced T-cell survival and apoptosis resistance, which is accompanied by GADD $45 \beta$ upregulation in these cells. We also found that IL-12 level is increased in recipient mice. As a result, Th1 response is upregulated in vivo, and CTL activity is enhanced, leading to augmented anti-tumor immunity [35].

Th1 cells play an important role in anti-tumor immune responses by producing a panel of cytokines such as IFN $\gamma$ and TNF $\alpha[1,2]$. IL-12 enhances Th1 cell polarization, leading to more IFN $\gamma$ and TNF $\alpha$ production. Through these cytokines, Th1 cells synergize with CTL and DC in anti-tumor immunity [4-9]. We have shown previously that immunization with attenuated activated autologous $\mathrm{T}$ cells leads to more IL-12 production. Here by using a more sensitive assay kit, we showed that our immunization procedure also increases IFN $\gamma$ and TNF $\alpha$ levels in the serum of immunized mice compared with naïve mice. Therefore, Th1 activity is enhanced by the immunization [35].

As Th1 cells belong to the CD4+ T cells, we intended to further examine the activation status of CD4+ T cells by analyzing their surface markers, such as CD62L and CD25. CD62L is usually expressed at a high level on resting $\mathrm{T}$ cells $[36,37]$, while CD25, the $\alpha$-chain of IL-2 receptor expressed upon T-cell activation, has been used as a marker for activated T cells [38-40]. The data showed CD62L+ Tcell population was reduced (and thus $\mathrm{CD} 62 \mathrm{~L}^{\text {low }}$ population was increased) in PBMC from immunized mice. We have shown previously that in the splenocytes, immunization actually led to an increase in CD62 L $+\mathrm{T}$ cells [35]. We think that this difference observed between peripheral and spleen $T$ cells may be due to their different respective microenvironments. In anti-tumor immunity, both CD4+ CD62 $\mathrm{L}^{\text {low }}$ and CD8+CD62 $\mathrm{L}^{\text {low }} \mathrm{T}$ cells are believed to play important roles [41, 42]. Our data suggested that immunization with attenuated activated autologous $\mathrm{T}$ cells increased CD62 $\mathrm{L}^{\text {low }} \mathrm{T}$ population, which is consistent with reports by other groups [41-43]. Given that CD25 is a component of IL-2 receptor which maintains T-cell proliferation and expansion after activation, and that Th1 activity is induced by our immunization, one would expect that the CD25+ population should be increased in CD4+ T cells from immunized mice. Somewhat surprisingly, the results from our experiments showed that CD25+ population actually 
decreased.

Recently, Treg have been identified as a subset of $\mathrm{CD} 4+\mathrm{CD} 25+\mathrm{T}$ cells and have been suggested to be responsible for inducing and maintaining peripheral tolerance as well as the negative regulation of tumor immunity [12-14, 24-26]. Given that our immunization induces anti-tumor immunity and decreases the $\mathrm{CD} 25+$ population in $\mathrm{CD} 4+\mathrm{T}$ cells, we hypothesized that the Treg population might be suppressed in immunized mice. To test this hypothesis, we examined $\mathrm{CD} 4+\mathrm{CD} 25+\mathrm{T}$ cells for the expression of Foxp3, a key marker for Treg. Our results showed that both the mRNA and protein levels of Foxp3 were significantly reduced in $\mathrm{CD} 4+\mathrm{CD} 25+\mathrm{T}$ cells obtained from immunized mice compared with that from naïve control. Functional analysis indicated that $\mathrm{CD} 4+\mathrm{CD} 25+\mathrm{T}$ cells from immunized mice exhibited a significantly lower ability to suppress the proliferation of activated T cells. These data support our hypothesis that the Treg population decreased in immunized mice. The suppressed Treg population and function likely contributed to the enhanced Th1 response in immunized mice, which in turn led to augmented antitumor immunity [26-29, 32, 33].

To gain insight into how our immunization led to reduced Treg population and function, we found that B220+ population was significantly higher in peripheral lymphocytes from immunized mice. This implicated that B cells were expanded by the immunization in vivo. Because B cell expansion usually leads to antibody production $[44,45]$, we examined the total IgG level in serum of immunized mice and found that its concentration was significantly higher (data not shown). In further analysis, we found that antiCD25 antibody was induced to a significantly higher level in recipient mice by immunization. These results suggested that immunization with attenuated activated autologous $\mathrm{T}$ cells could induce anti-CD25 antibody production, which might be responsible for the observed decrease of Treg population.

To address why our immunization would stimulate the production of anti-CD25 antibody, we examined the activated autologous $\mathrm{T}$ cells used in immunization for their surface antigen expression, and found that CD25 was the dominantly expressed molecule on these cells. From these data, we suggest that immunization with these cells would present an abundant source of CD25 molecules, which could stimulate B cell expansion and the production of anti-CD25 antibody in vivo.

It is known that $\mathrm{CD} 4+\mathrm{CD} 25+\mathrm{T}$ are a heterogeneous population consisting of activated CD4+ T cells and Treg [12-14]. To further test the hypothesis that the high level of anti-CD25 antibody in immunized mice is responsible for the impeded Treg function, serum adoptive transfer experiments were performed, where serum was isolated from immunized mice and injected i.v. into naïve recipient mice. The results demonstrated that CD4+CD25+Foxp3+ T cells in recipient mice decreased and dropped to the lowest level at day 2 after the serum transfer. Functional assays confirmed that $\mathrm{CD} 4+\mathrm{CD} 25+\mathrm{T}$ cells from the recipient mice showed impeded Treg function as their ability to inhibit activated T-cell proliferation is significantly impaired.

It is well known that Treg are responsible for inducing and maintaining peripheral tolerance and the negative regulation of immunity [24, 25]. Treg have been suggested to play an important immunopathological role in human cancer by 'lowering' the intrinsic T-cell immunity towards tumor-associated antigen, resulting in tumor immune evasion [14, 27-29]. Some reports have indicated that Treg depletion can be useful in tumor biotherapy $[32,33]$. Treg depletion would eliminate immune-suppression mediated by Treg leading to enhanced T-cell activity. We show here that immunization with attenuated activated autologous $\mathrm{T}$ cells was able to markedly decrease Treg population and function in vivo, which we think at least partially contributed to the enhanced Th1 activity and anti-tumor immunity in immunized mice. Indeed, studies in mice with tumor inoculation after the immunization showed that inhibition of tumor growth is accompanied by Treg downregulation (our unpublished data).

In conclusion, our results demonstrate for the first time that immunization with attenuated activated autologous $\mathrm{T}$ cells evokes anti-CD25 antibody production and leads to impeded CD4+CD25+Foxp3+ Treg function in vivo. We suggest that the dampened Treg function likely contributes to enhanced Th1 response in immunized mice and is at least part of the mechanism underlying the boosted anti-tumor immunity.

\section{Acknowledgments}

This work was supported by National Natural Science Foundation of China (No. 30671945), Science and Technology Commission of Shanghai Municipality (Nos. 06JC14044，05ZR14055，054319928，04DZ14902), Shanghai Municipal Education (No. 05BZ26), Shanghai Leading Academic Discipline Project (T0206) and Science Foundation of Shanghai Institute of Immunology (No. 07A04, to Ningli Li).

\section{References}

1 Schmitz-Winnenthal FH, Volk C, Z'graggen K, et al. High frequencies of functional tumor-reactive $\mathrm{T}$ cells in bone marrow and blood of pancreatic cancer patients. Cancer Res 2005; 65:10079-10087.

2 Hokey DA, Larregina AT, Erdos G, et al. Tumor cell loaded type1 polarized dendritic cells induce Th1-mediated tumor immunity. 
Cancer Res 2005; 65:10059-10067.

3 Morishima N, Owaki T, Asakawa M, et al. Augmentation of effector $\mathrm{CD} 8+\mathrm{T}$ cell generation with enhanced granzyme $\mathrm{B}$ expression by IL-27. J Immunol 2005; 175:1686-1693.

4 Kalams SA, Walker BD. The critical need for CD4 help in maintaining effective cytotoxic T lymphocyte responses. J Exp Med 1998; 188:2199-2204.

5 van Gisbergen KP, Aarnoudse CA, Meijer GA, et al. Dendritic cells recognize tumor-specific glycosylation of carcinoembryonic antigen on colorectal cancer cells through dendritic cell-specific intercellular adhesion molecule-3-grabbing nonintegrin. Cancer Res 2005; 65:5935-5944.

6 Voigtlander C, Rossner S, Cierpka E, et al. Dendritic cells matured with TNF can be further activated in vitro and after subcutaneous injection in vivo which converts their tolerogenicity into immunogenicity. J Immunother 2006; 29:407-415.

7 Muthian G, Raikwar HP, Johnson C, et al. COX-2 inhibitors modulate IL-12 signaling through JAK-STAT pathway leading to Th1 response in experimental allergic encephalomyelitis. J Clin Immunol 2006; 26:73-85.

8 Trinchieri G. Interleukin-12 and the regulation of innate resistance and adaptive immunity. Nat Rev Immunol 2003; 3:133146.

9 Kumaraguru U, Suvas S, Biswas PS, Azkur AK, Rouse BT. Concomitant helper response rescues otherwise low avidity CD8+ memory CTLs to become efficient effectors in vivo. J Immunol 2004; 172:3719-3724.

10 Kass R, Agha J, Bellone S, et al. In vitro induction of tumorspecific HLA class I-restricted CD8+ cytotoxic T lymphocytes from patients with locally advanced breast cancer by tumor antigen-pulsed autologous dendritic cells. J Surg Res 2003; 112:189-197.

11 Xia D, Moyana T, Xiang J. Combinational adenovirus-mediated gene therapy and dendritic cell vaccine in combating well-established tumors. Cell Res 2006; 16:241-259

12 Finn OJ. Cancer vaccines: between the idea and the reality. Nature Rev Immunol 2003; 3:630-641.

13 Khong HT, Restifo NP. Natural selection of tumor variants in the generation of 'tumor escape' phenotypes. Nature Immunol 2002; 3:999-1005.

14 Walker LS, Chodos A, Eggena M, Dooms H, Abbas AK. Antigendependent proliferation CD4+CD25+ regulatory $\mathrm{T}$ cells in vivo. J Exp Med 2003; 198:249-258.

15 Yazawa T, Ito T, Kamma H, et al. Complicated mechanisms of class II transactivator transcription deficiency in small cell lung cancer and neuroblastoma. Am J Pathol 2002; 161:291-300.

16 Yazawa T, Kamma H, Fujiwara M, et al. Lack of class II transactivator causes severe deficiency of HLA-DR expression in small cell lung cancer. J Pathol 1999; 187:191-199.

17 Francesca Fallarino, Ursula Grohmann, Roberta Bianchi, et al. Th1 and Th2 cell clones to a poorly immunogenic tumor antigen initiate CD8+ T cell-dependent tumor eradication in vivo. J Immunol 2000; 165:5495-5501.

18 Kalams SA, Walker BD. The critical need for CD4 help in maintaining effective cytotoxic T lymphocyte responses. J Exp Med 1998; 188:2199.

19 Andersen MH, Ostergaard Pedersen L, Capeller B, et al. Spontaneous cytotoxic $\mathrm{T}$ cell responses against surviving-derived MHC class I-restricted T cell epitopes in situ as well as ex vivo in cancer patients. Cancer Res 2001; 61:5964-5971.

20 Cinatl JJ, Scholz M, Doerr HW. Role of tumor cell immune escape mechanisms in cytomegalovirus-mediated oncomodulation. Med Res Rev 2005; 25:167-185.

21 Surman D, Dudley ME, Overwijk WW, Restifo NP. Cutting edge: CD4+ T cell control of CD8+ T cell reactivity to a model tumor antigen. J Immunol 2000; 164:562-565.

22 Weiping Zou. Immunosuppressive networks in the tumour environment and their therapeutic relevance. Nat Rev Cancer 2005; 5:263-274.

23 Dunn GP, Bruce AT, et al. Cancer immunoediting: from immunosurveillance to tumor escape. Nat Immunol 2002; 3:991-998.

24 Maria A Curotto de Lafaille, Juan J Lafaille, et al. CD4+ regulatory $\mathrm{T}$ cells in autoimmunity and allergy. Curr Opin Immunol 2002; 14:771-778.

25 Harald von Boehmer. Mechanisms of suppression by suppressor T cells. Nat Immunol 2005; 6:338-344.

26 Turk MJ, Guevara-Patino JA, Rizzuto, et al. Concomitant tumor immunity to a poorly immunogenic melanoma is prevented by regulatory T cells. J Exp Med 2004; 200:771-782.

27 Curiel TJ, Coukos G, Zou L, et al. Specific recruitment of regulatory $\mathrm{T}$ cells in ovarian carcinoma fosters immune privilege and predicts reduced survival. Nat Med 2004; 10: 942-949.

28 Lars A Ormandy, Tina Hillemann, Heiner Wedemeyer, et al. Increased populations of regulatory $\mathrm{T}$ cells in peripheral blood of patients with hepatocellular carcinoma. Cancer Res 2005; 65:2457-2464.

29 Sakaguchi S. Naturally arising Foxp3-expressing CD25+CD4+ regulatory $\mathrm{T}$ cells in immunological tolerance to self and nonself. Nat Immunol 2005; 6:345-352.

30 Leach DR, Krummel MF, Allison JP. Enhancement of antitumor immunity by CTLA-4 blockade. Science 1996; 271:17341736.

31 Hurwitz AA, Yu TF, et al. CTLA-4 blockade synergizes with tumor-derived granulocyte macrophage colony-stimulating factor for treatment of an experimental mammary carcinoma. Proc Natl Acad Sci USA 1998; 95:10067-10071.

32 Shozaburo Onizuka, Isao Tawara, Jun Shimizu, et al. Tumor Rejection by in vivo administration of anti-CD25 (interleukin-2 receptor a) monoclonal antibody. Cancer Res 1999; 59:31283133.

33 Jun Shimizu, Sayuri Yamazaki, Shimon Sakaguchi. Induction of tumor immunity by removing $\mathrm{CD} 25+\mathrm{CD} 4+\mathrm{T}$ cells: a common basis between tumor immunity and autoimmunity. J Immunol 1999; 163:5211-5218.

34 Sutmuller RP, et al. Synergism of cytotoxic T lymphocyte associated antigen 4 blockade and depletion of $\mathrm{CD} 25+$ regulatory $\mathrm{T}$ cells in antitumor therapy reveals alternative pathways for suppression of autoreactive cytotoxic T lymphocyte responses. J Exp Med 2001; 194:823-832.

35 Li Wang, Fang Du, Qi Cao, et al. Immunization with autologous T cells enhances in vivo anti-tumor immune responses accompanied by up-regulation of GADD45 $\beta$. Cell Res 2006; 16:702-712.

36 Lu LF, Gondek DC, Scott ZA, Noelle RJ. NF kappa B-inducing kinase deficiency results in the development of a subset of regulatory $\mathrm{T}$ cells, which shows a hyperproliferative activity upon glucocorticoid-induced TNF receptor family-related gene stimulation. J Immunol 2005; 175:1651-1167.

37 Wang LX, Chen BG, Plautz GE. Adoptive immunotherapy of 
advanced tumors with CD62 L-selectin(low) tumor-sensitized $\mathrm{T}$ lymphocytes following ex vivo hyperexpansion. J Immunol 2002; 169:3314-3320.

38 Hannier S, Bitegye C, Demotz S. Early events of TCR signaling are distinct in human Th1 and Th2 cells. J Immunol 2002; 169:1904-1911.

39 Kim HP, Leonard WJ. The basis for TCR-mediated regulation of the IL-2 receptor alpha chain gene: role of widely separated regulatory elements. EMBO J 2002 21:3051-3059.

40 von Geldern M, Simm B, Braun M, et al. TCR-independent cytokine stimulation induces non-MHC-restricted T cell activity and is negatively regulated by HLA class I. Eur J Immunol 2006; 36:2347-2358.

41 Hiura T, Kagamu H, Miura S, et al. Both regulatory T cells and antitumor effector $T$ cells are primed in the same draining lymph nodes during tumor progression. J Immunol 2005; 175:5058-
5066.

42 Peng L, Kjaergaard J, Plautz GE, et al. Helper-independent, L-selectinlow CD8+ T cells with broad anti-tumor efficacy are naturally sensitized during tumor progression. J Immunol 2000; 165:5738-5749.

43 Enarsson K, Johnsson E, Lindholm C, et al. Differential mechanisms for $\mathrm{T}$ lymphocyte recruitment in normal and neoplastic human gastric mucosa. Clin Immunol 2006; 118:24-34.

44 Matsumura Y, Byrne SN, Nghiem DX, Miyahara Y, Ullrich SE. A role for inflammatory mediators in the induction of immunoregulatory B cells. J Immunol 2006; 177:4810-4817.

45 Strutt TM, Uzonna J, McKinstry KK, Bretscher PA. Activation of thymic $\mathrm{T}$ cells by $\mathrm{MHC}$ alloantigen requires syngeneic, activated CD4+ T cells and B cells as APC. Int Immunol 2006; 18:719-728 